\title{
EMC3 coordinates surfactant protein and lipid homeostasis required for respiration
}

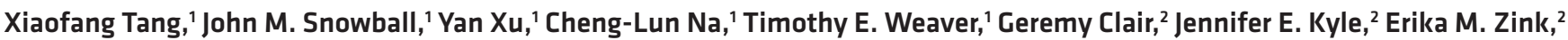 \\ Charles Ansong, ${ }^{2}$ Wei Wei, ${ }^{3}$ Meina Huang, ${ }^{3}$ Xinhua Lin, ${ }^{3,4}$ and Jeffrey A. Whitsett ${ }^{1}$ \\ 'Divisions of Neonatology, Perinatal and Pulmonary Biology, Perinatal Institute, Cincinnati Children's Hospital Medical Center, Cincinnati, Ohio, USA. 'Biological Sciences Division, Pacific Northwest National \\ Laboratory, Richland, Washington, USA. 'State Key Laboratory of Genetic Engineering, Institute of Cenetics, Collaborative Innovation Center of Genetics and Development, School of Life Sciences, Fudan \\ University, Shanghai, China. `Division of Developmental Biology, Perinatal Institute, Cincinnati Children's Hospital Medical Center, Cincinnati, Ohio, USA.
}

\begin{abstract}
Adaptation to respiration at birth depends upon the synthesis of pulmonary surfactant, a lipid-protein complex that reduces surface tension at the air-liquid interface in the alveoli and prevents lung collapse during the ventilatory cycle. Herein, we demonstrated that the gene encoding a subunit of the endoplasmic reticulum membrane complex, EMC3, also known as TMEM111 (Emc3/Tmem111), was required for murine pulmonary surfactant synthesis and lung function at birth. Conditional deletion of Emc3 in murine embryonic lung epithelial cells disrupted the synthesis and packaging of surfactant lipids and proteins, impaired the formation of lamellar bodies, and induced the unfolded protein response in alveolar type 2 (AT2) cells. EMC3 was essential for the processing and routing of surfactant proteins, SP-B and SP-C, and the biogenesis of the phospholipid transport protein ABCA3. Transcriptomic, lipidomic, and proteomic analyses demonstrated that EMC3 coordinates the assembly of lipids and proteins in AT2 cells that is necessary for surfactant synthesis and function at birth.
\end{abstract}

\section{Introduction}

Pulmonary surfactant is a complex mixture of lipids and associated proteins synthesized by alveolar type 2 (AT2) cells in the peripheral lung. Surfactant is required at birth and throughout postnatal life to reduce surface tension at the air-liquid interface in the alveoli. Lack of surfactant causes atelectasis and respiratory distress syndromes in premature infants (RDS) and adults (ARDS) (1). Surfactant proteins, and a phospholipid transport protein, $\mathrm{ABCA}$, are required for the packaging of phospholipids into specialized organelles termed lamellar bodies (LBs) by AT2 cells and for the function of surfactant in the alveoli. Mutations in the ABCA3, SFTPB, and SFTPC genes cause severe lung disease in newborn infants and children, usually presenting with respiratory failure soon after birth ( $A B C A 3$ and SFTPB) or in infancy (SFTPC) (1-4). During transit from the ER to LBs, surfactant proteins B and C (SP-B and SP-C) are folded, proteolytically processed, and trafficked from the Golgi to multivesicular bodies, and ultimately stored with surfactant phospholipids in LBs. The fully processed SP-B and SP-C are secreted together with phospholipids onto alveolar surfaces to reduce surface tension and prevent lung collapse during the ventilatory cycle (5). ABCA3, a multipass transmembrane protein, is routed to the limiting membrane of the LB where it is required for transporting phosphatidylcholine (PC) and phosphatidylglycerol (PG) into the organelle (6, 7). Cellular mechanisms coordinating synthesis and assembly of phospholipids, surfactant proteins, and ABCA3 required for normal surfactant homeostasis remain incompletely understood.

Conflict of interest: The authors have declared that no conflict of interest exists. Submitted: April 4, 2017; Accepted: September 26, 2017

Reference information: / Clin Invest. 2017;127(12):4314-4325.

https://doi.org/10.1172/JCI94152.
EMC3, encoded by the mouse Tmem111 gene, is a subunit in the highly conserved ER membrane protein complex termed the EMC. The EMC was first identified in Saccharomyces cerevisiae as a 6-subunit transmembrane protein complex required for protein folding in the ER (8). Loss of EMC subunits in yeast causes accumulation of misfolded membrane proteins and induction of the unfolded protein response (UPR). Emc1, Emc2, and Emc3 form a complex with ER-associated degradation (ERAD) pathway components Ubac2 and Derlin-2, indicating a close link between the EMC and ERAD (9). Moreover, EMCs are essential for the assembly of several multipass membrane proteins including nicotinic acetylcholine receptors (AChRs) in Caenorhabditis elegans $(10,11)$ and rhodopsin in Drosophila (12) and Danio (13). Despite its involvement in important cellular processes, the functions of Emc3 in vertebrate development and organ function are unclear.

Acute respiratory failure and chronic interstitial lung disease are caused by mutations in $A B C A 3, S F T P B$, and SFTPC that produce misfolded proteins, inducing UPR and causing AT2 cell injury (1-4, 14-16). The folding, proteolytic processing, and intracellular transport of $A B C A 3$ and surfactant proteins through the ER are critical for the production of pulmonary surfactant. In this report, we tested whether EMC3 plays a role in the assembly of surfactant lipids and proteins in AT2 cells in the neonatal mouse lung. We show that, in spite of normal lung morphogenesis, deletion of $E m c 3$ in embryonic lung epithelial cells led to respiratory distress and neonatal lethality related to surfactant deficiency. Furthermore, we demonstrate that EMC3 controls the processing and routing of surfactant proteins SP-B and SP-C as well as the biogenesis of the phospholipid transporter protein ABCA3. Therefore, we have identified EMC3 as a critical component of an ER complex that mediates the processing and trafficking of surfactant- 
A $\underset{\text { Emc }^{\mathrm{TG} /+}}{\text { Targeted allele }}$

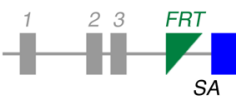
lacZ $P_{p A}^{10 x P}$ neo FRT $\operatorname{loxP} 45 \operatorname{loxP} 6$ $p$

B

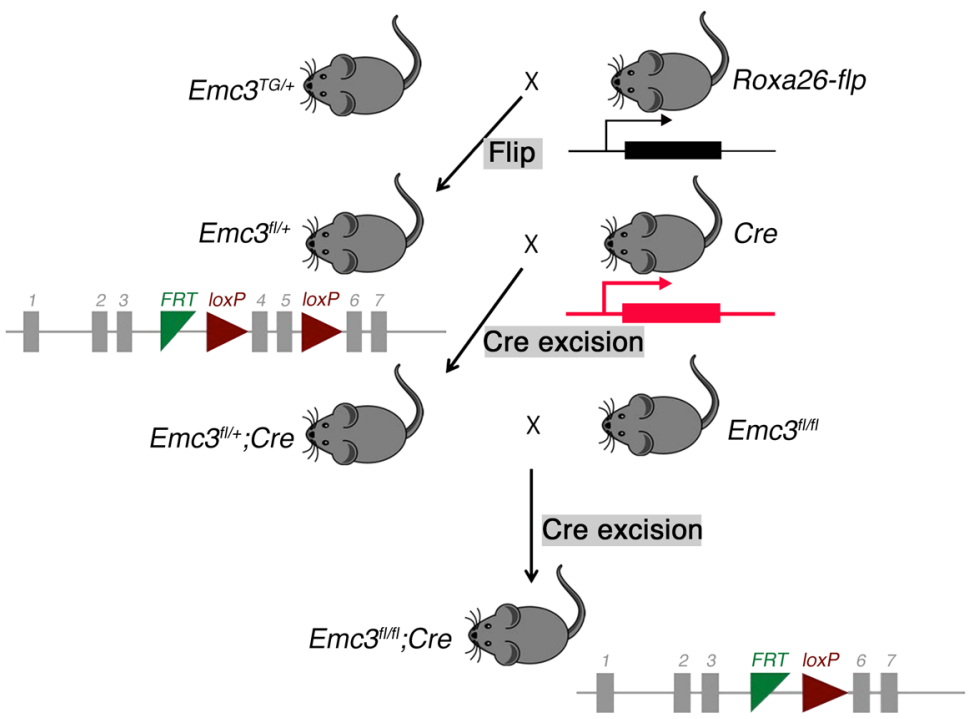

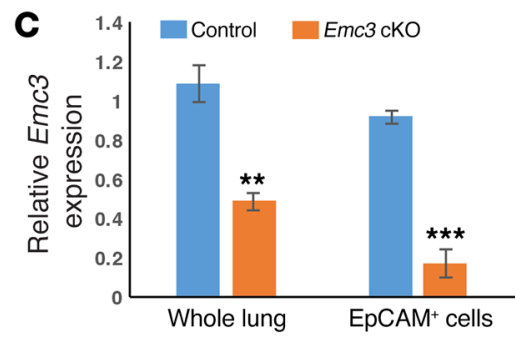

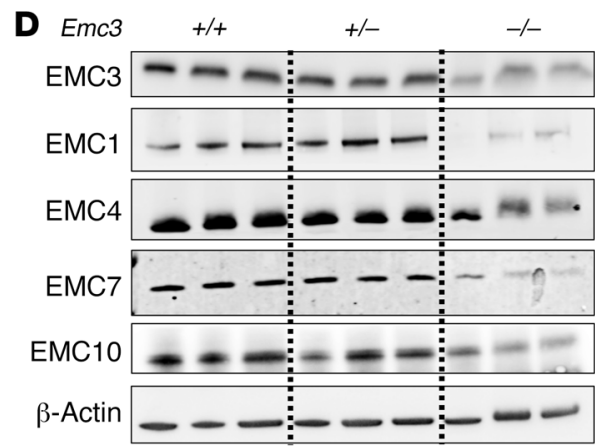

Figure 1. Conditional deletion of Emc3 with Shh-Cre. (A) Design of the Emc3 gene targeted allele is shown. The critical exons 4 and 5 are flanked by loxP sites. (B) The breeding strategy to generate $E m c 3$ conditional knockout mice is shown. The Emc3 conditional allele was created after flippase recombination. Subsequently, the Shh-Cre promoter-driven targeting cassette deletes exons 4 and 5. (C) qPCR of Emc3 RNA isolated from E18.5 control and Emc3-cKO whole-lung tissue or EpCAM+ ${ }^{+}$sorted lung epithelial cells. Emc3 RNA was significantly decreased in homozygous Emc3-cKO mice. Data are the mean \pm SEM. ${ }^{* *} P<0.01$, ${ }^{* *} P<0.001$ using unpaired, 2-tailed Student's $t$ test. $n=4$ /group. (D) Whole-lung lysates were prepared from control and mutant mice at E18.5. Western blots were performed using indicated EMC and $\beta$-actin antibodies. Levels of all EMC subunits tested were decreased by deletion of Emc3.

associated proteins and lipids by AT2 cells and is required for lung function and respiration at birth.

\section{Results}

Conditional deletion of Emc3 in respiratory epithelium causes surfactant deficiency and neonatal respiratory failure. To assess the role(s) of EMC3 in lung epithelial cells, an Emc3 $3^{f / f l}$ allele was produced and mated to Shh-Cre-transgenic mice to delete Emc3 in respiratory epithelial cells prior to birth (Figure 1, A and B). While ShhCre;Emc ${ }^{f /+}$ mice survived normally, all Shh-Cre;Em $c^{f l / f l}$ mice died of respiratory failure immediately after birth (Table 1). Cre-mediated excision in Shh-Cre;Emct/fl (referred to as Emc3 cKO hereafter) mice reduced $E m c 3$ mRNA levels in whole-lung homogenates and isolated $\mathrm{EpCAM}^{+}\left(\mathrm{CD} 326^{+}\right)$epithelial cells at E18.5, the day before birth (Figure 1C and Supplemental Figure 1A; supplemental material available online with this article; https:// doi.org/10.1172/JCI94152DS1). Western blot analysis demonstrated decreased EMC3 protein levels (Figure 1D). Deletion of Emc3 decreased the other EMC proteins we examined including EMC1, 4, 7, and 10 but did not influence their RNAs, indicating the essential role of EMC3 in stabilizing the entire EMC complex (Figure 1D, and Supplemental Figure 1, A and C). Without any observable malformations, Emc3-deficient pups remained cyanotic and failed to inflate their lungs in spite of vigorous respiratory efforts, while control $\left(\mathrm{Emc}^{+/+}\right)$littermates were well oxygenated and survived (Figure 2, A-D). Histological analysis of lung sections at E18.5 showed no abnormalities in lung morphogenesis (Figure 2, E and F). Differentiation of AT1 cells, indicated by podoplanin (PDPN), AGER, and HOPX staining, was undisturbed (Supplemental Figure 2), supporting the concept that defective surfactant homeostasis rather than a developmental delay may underlie the respiratory failure seen in the Emc3-cKO mice. Ultrastructure of AT2 cells in Emc3-mutant lungs lacked normal LBs and instead contained small, poorly lamellated vesicles, while the ultrastructure of other intracellular organelles was unchanged (Figure 2, G and $\mathrm{H}$, and Table 2). Given the essential function of LBs in surfactant packaging, secretion, and function, the abnormal LB morphology associated with Emc3 deletion demonstrated that EMC3 played an important role in surfactant biosynthesis before birth.

\section{Table 1. Postnatal death in Emc3-ckO mice}

\begin{tabular}{|c|c|c|c|c|}
\hline \multirow[b]{2}{*}{ Progeny genotype } & \multicolumn{4}{|c|}{ Male Shh-Cre;Emc $3^{f / /+} \times$ Female $E m c 3^{f / f f l}$} \\
\hline & $E m c 3^{f / /+}$ & $E m c^{f / f / f l}$ & Shh-Cre; Emc $3^{f / /+}$ & Shh-Cre; Emc $3^{f / / f l}$ \\
\hline Expected & $25 \%$ & $25 \%$ & $25 \%$ & $25 \%$ \\
\hline Progeny at weaning $(n=80)$ & $32.5 \%(26)$ & $35.0 \%(28)$ & $32.5 \%(26)$ & 0 \\
\hline Progeny at E18.5 $(n=149)$ & $21.5 \%(32)$ & $23.5 \%(35)$ & $28.2 \%(42)$ & $26.8 \%(40)$ \\
\hline
\end{tabular}

The genotypes and numbers of progeny generated are listed. Of 80 pups genotyped at weaning, no Shh$\mathrm{Cre} ; \mathrm{Emc} \mathrm{3}^{\mathrm{fl} / \mathrm{fl}}$ mice were observed. For progeny analyzed at E18.5, the percentage of each genotype was consistent with the expected Mendelian ratio. 


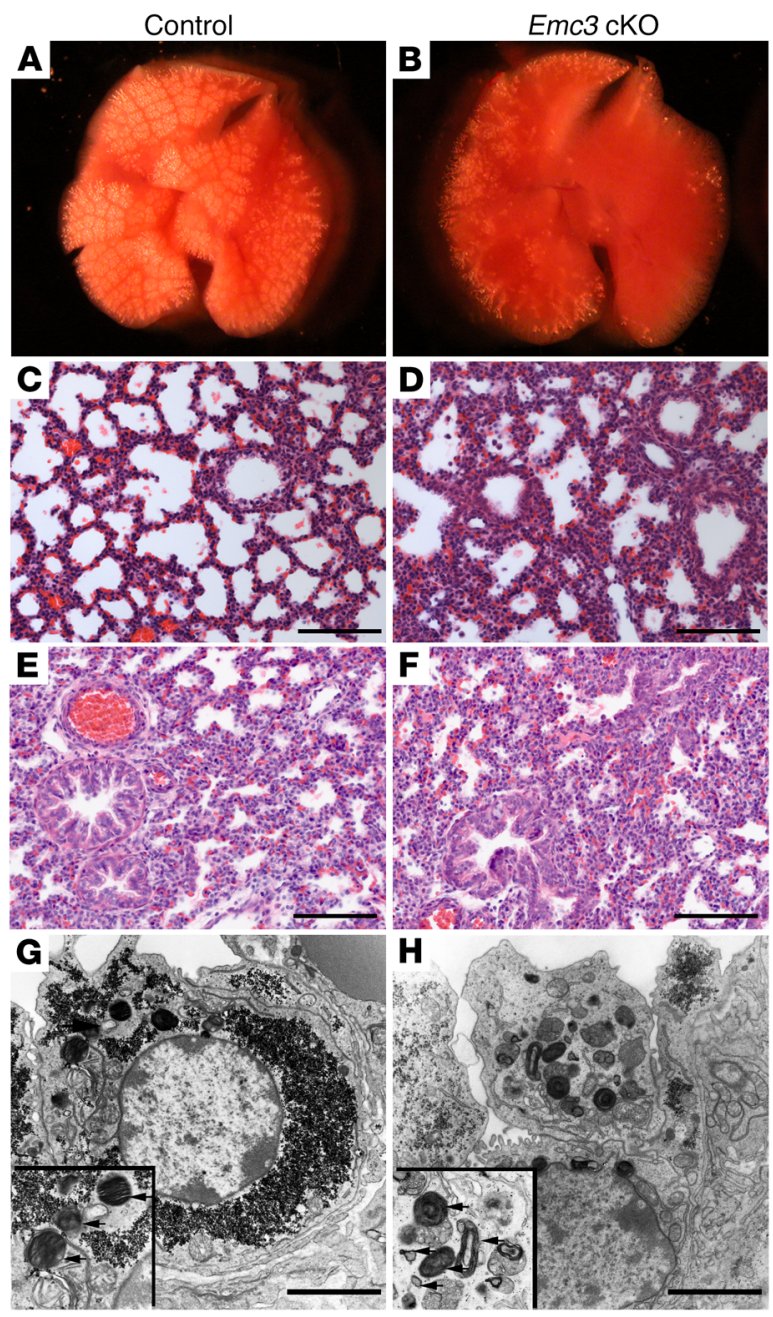

Emc3 deficiency leads to decreased ABCA3 and abnormal processing and trafficking of surfactant-associated proteins. Deleterious mutations in SFTPB or ABCA3 impair LB formation and surfactant production in both humans and mice $(1,17,18)$. We therefore assessed components of the surfactant system required for normal postnatal respiration. While the expression of $S f t p b, S f t p c$, and Abca3 RNAs remained unaltered (Supplemental Figure 1B), the protein levels or distribution of ABCA3, SP-B, and SP-C were disrupted in the absence of EMC3. A marked reduction in ABCA3 protein levels was observed in the peripheral lung of Emc3cKO mice as assessed by immunostaining (Figure 3, A and B), immunoblotting (Figure 3G), and immunogold ultrastructural labeling (Supplemental Figure 3, $A$ and $B$ ). The precursor of SP-B protein (proSP-B) accumulated and was abnormally secreted, while the active $8-\mathrm{kDa}$ SP-B peptide (mSP-B) was nearly absent (Figure 3, A, B, and H), demonstrating a lack of proteolytic pro-
Figure 2. Surfactant deficiency and neonatal respiratory failure in Emc3-cKO mice. (A and B) Lungs from control and Emc3-cKO mice (PO) are shown, demonstrating diffuse atelectasis after deletion of Emc3. (C and D) Hematoxylin/eosin-stained sections show normal lung morphogenesis but collapsed airspaces in Emc3-cKO mice at PO. Scale bars: $100 \mu \mathrm{m}$. (E and F) Hematoxylin/eosin-stained sections show normal lung histology in Emc3cKO mice at E18.5, the day before birth. Scale bars: $100 \mu \mathrm{m}$. (G and H) Electron microscopic images identify lamellar body-like inclusions (arrows) in control (C) and Emc3-deleted (H) AT2 cells at E18.5. AT2 cells from Emc3cKO mice lacked normal lamellar bodies and contained abnormal, smaller, and heterogeneous lamellar body-like inclusions. Scale bars: $2 \mu \mathrm{m}$.

cessing and misrouting of proSP-B. ProSP-C staining was increased in the cytoplasm of AT2 cells, while the mature 3-kDa SP-C peptide (mSP-C) was abnormally colocalized with proSP-C and was not secreted into the alveolar spaces (Figure 3, C, D, and H). To determine intracellular distributions of proSP-B and proSP-C, we performed coimmunofluorescence staining with LAMP-1, which stains lysosome-related organelles, including LBs, multivesicular bodies (MVBs), and lysosomes. ProSP-B and proSP-C were colocalized with LAMP-1 in AT2 cells of Emc3-cKO mice (Figure 3, E and $\mathrm{F}$ ), indicating retention of unprocessed precursors. Consistent with these findings, immunogold-labeled proSP-B and proSP-C were increased in the MVBs of Emc3-deficient AT2 cells (Supplemental Figure 3, C-F). Altogether, our data demonstrate that EMC3 is required for stabilization of the ABCA3 protein and for the normal processing and trafficking of SP-B and SP-C, processes that are necessary for surfactant function.

EMC3 interacts with $A B C A 3$ and controls $A B C A 3$ stability. Since EMC3 was previously shown to influence folding of multipass transmembrane proteins, including AChRs and rhodopsin (10-13), we sought to test whether EMC interacts with ABCA3 to influence its stability. To model AT2 cells in vivo, we utilized MLE15 cells (mouse lung epithelial cell line transformed with SV40 large T-antigen) that express both $\mathrm{ABCA} 3$ and the surfactant proteins SP-B and SP-C (19). Consistent with its function as a subunit of the EMC complex, endogenous EMC3 was localized in the $\mathrm{ER}$, as revealed by its colocalization with the ER marker, protein disulfide isomerase (PDI) (Supplemental Figure 4A). Expression of Flag-tagged ABCA3 recruited endogenous EMC3 to intracellular compartments positive for LAMP-1, indicating the interaction and cotransport of ABCA3 and EMC3 from the ER to MVBs/ LBs (Supplemental Figure 4, B and C). To further characterize the interaction between EMC3 and ABCA3, a construct encod- 

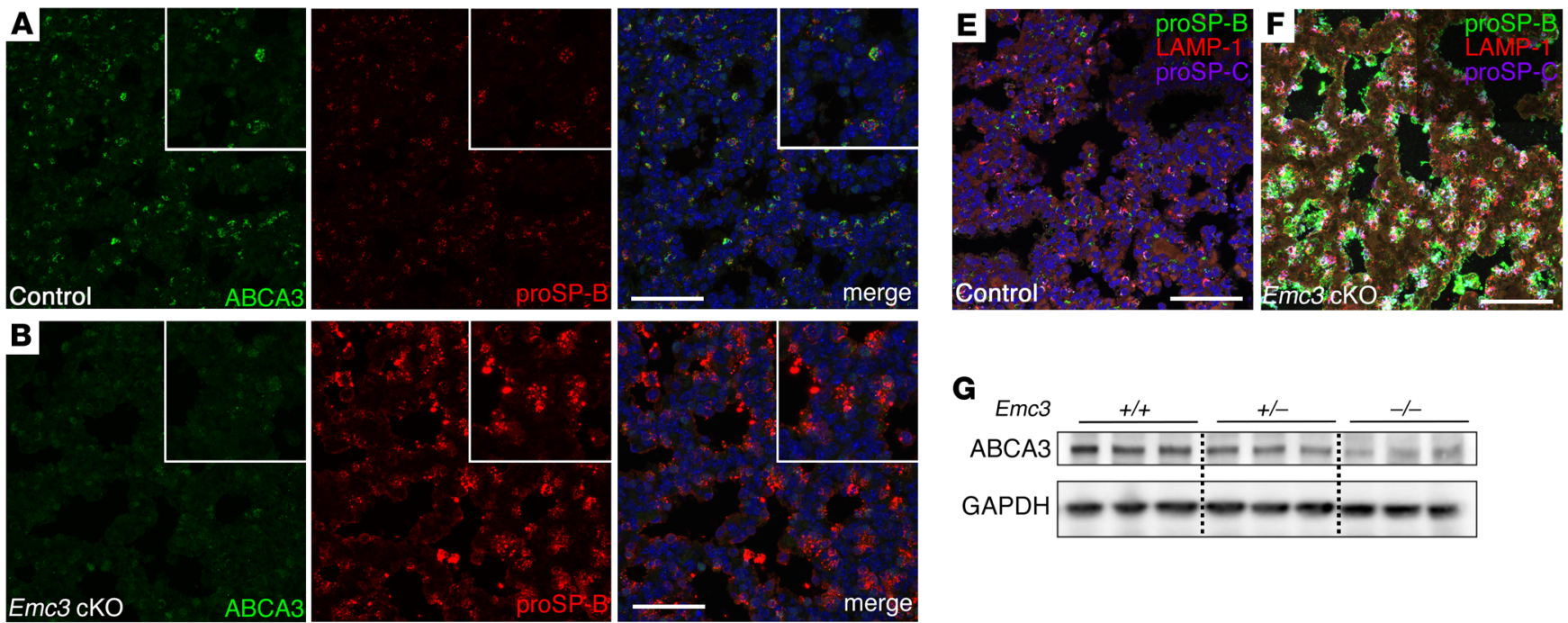

\section{G}
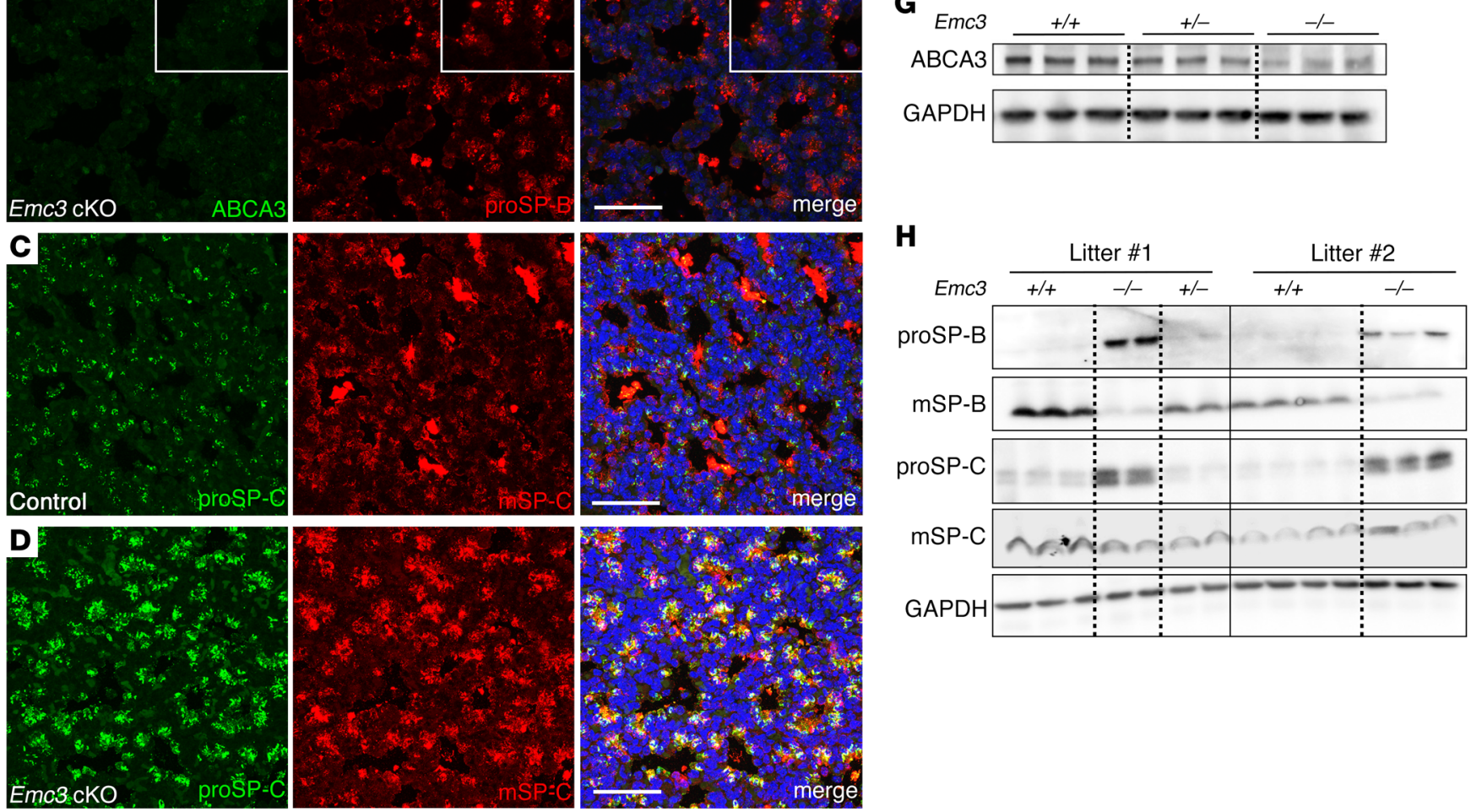

Figure 3. EMC3 is required for normal processing and trafficking of surfactant-associated proteins. (A and B) E18.5 control and Emc3-cKO lung sections were stained for ABCA3 and proSP-B. ABCA3 staining was reduced and proSP-B increased in AT2 cells from Emc3-cKO mice. ProSP-B was abnormally present in peripheral lung saccules in Emc3-cKO mice, indicating its abnormal trafficking. (C and D) E18.5 control and Emc3-cKO lung sections were stained for proSP-C and mature SP-C (mSP-C). Both proSP-C and mSP-C accumulated in the cytoplasm and mSP-C was not secreted from Emc3-cKO AT2 cells. (E and F) E18.5 control and Emc3-cKO lung sections were stained for proSP-B, proSP-C, and LAMP-1, a marker for lysosome-related organelles. Intracellularly retained proSP-B and proSP-C colocalized with LAMP-1 in Emc3-cKO AT2 cells. (G) Western blotting of whole-lung lysates demonstrated decreased ABCA3 in Emc3-cKO lung. (H) Western blotting of whole-lung lysates showing the accumulation of proSP-B and proSP-C, and lack of mature SP-B (mSP-B) in Emc3-cKO lung. Scale bars: $50 \mu \mathrm{m}$.

ing EMC3 was produced with an N-terminal c-Myc tag. When transfected into MLE-15 cells, Myc-EMC3 acted the same way as endogenous EMC3; it was readily detected in the ER, where it was colocalized with calnexin (Supplemental Figure 5A). In MLE15 cells coexpressing Myc-EMC3 and ABCA3-Flag, the 2 proteins were primarily colocalized with LAMP-1, but not with calnexin, in intracellular compartments (Figure 4, A and B). Furthermore, Myc-EMC3 and ABCA3-Flag coimmunoprecipitated (co-IP) with other endogenous EMC subunits, including EMC1 and EMC4 (Figure $4 \mathrm{C}$ and Supplemental Figure 4D), indicating that ABCA3 and EMC proteins formed a complex in MLE-15 cells. RNAi-mediated knockdown of Emc1, Emc3, or Emc4 destabilized the complex (Figure $4 \mathrm{D}$ ), demonstrating that they function together. Consistent with Emc3-cKO defects, efficient knockdown of the EMC complex by Emc3 siRNA and Emc4 siRNA decreased the stability of ABCA3Flag in MLE-15 cells (Figure 4D). Taken together, these data support the concept that EMC3 interacts with $\mathrm{ABCA} 3$ to regulate its biogenesis. With similar assays, we were not able to detect direct interactions between proSP-C and Myc-EMC3 (Supplemental Figure 5, B and C), implying a distinct mechanism underlying the control of processing and trafficking of this surfactant protein by EMC3. Consistent with this finding, changes in the profiles from whole-lung mRNA in Emc3-cKO mice were distinct from those previously shown in Abca3-deficient lung (18) (Supplemental Table 1), supporting an independent role of EMC3 in surfactant biogenesis.

Emc3 deletion induces the UPR in AT2 cells. AT2 cell function is intimately related to the synthesis and processing of surfactant proteins and lipids. Mutations in SFTPC, SFTPB, ABCA3, and genes 

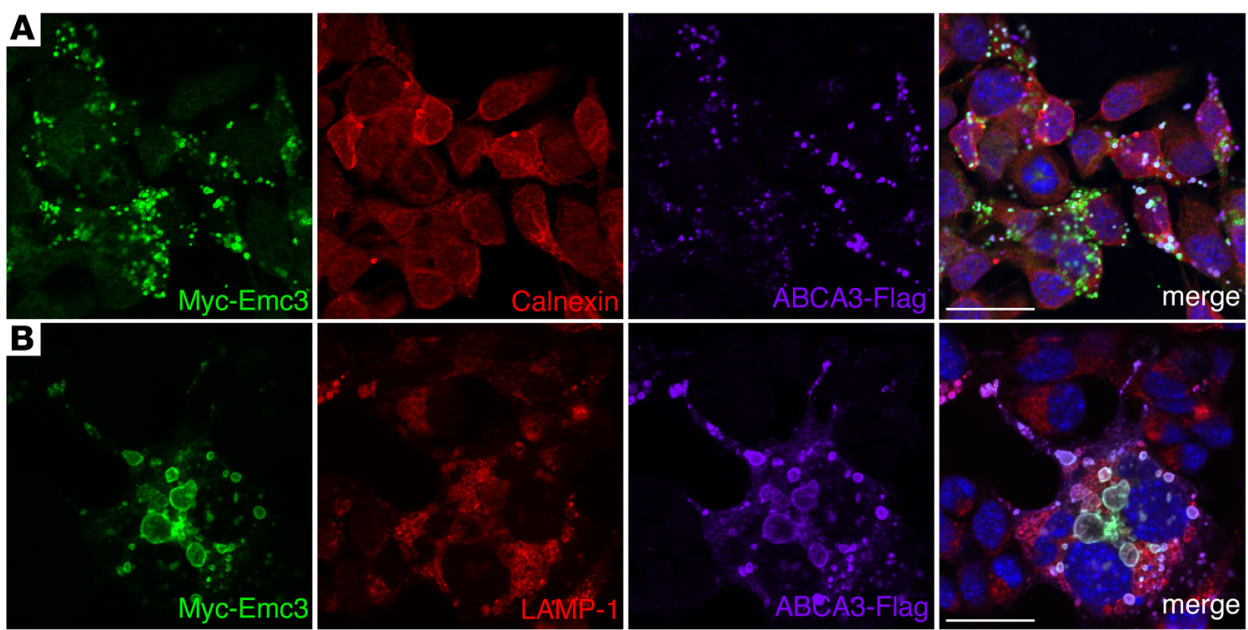

C

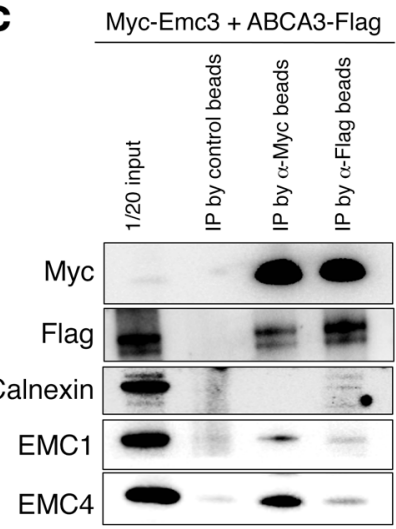

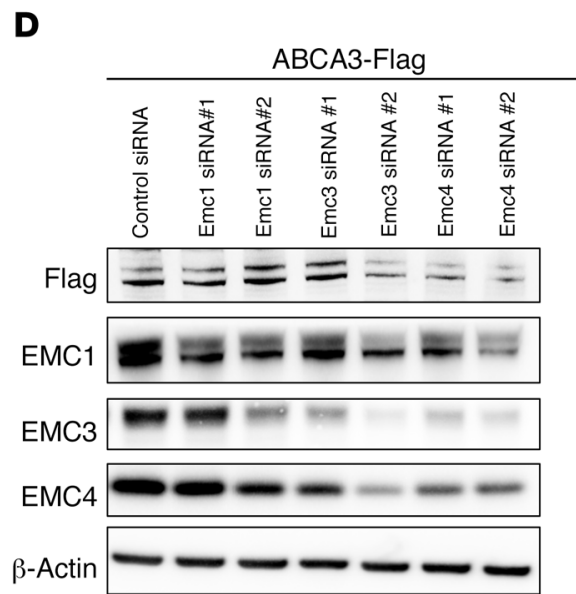

Figure 4. EMC3 interacts with $A B C A 3$ and regulates ABCA3 stability in MLE-15 cells. (A and B) MLE-15 cells were cotransfected with ABCA3-Flag and Myc-EMC3 and immunostained 48 hours later. Myc-EMC3 and $A B C A 3-F l a g$ were detected primarily in LAMP-1+ organelles. Scale bars: $25 \mu \mathrm{m}$. (C) MLE-15 cells cotransfected with ABCA3-Flag and Myc-EMC3 were lysed 48 hours after transfection and equal amounts of lysate were immunoprecipitated by control beads or beads conjugated with anti-Myc ( $\alpha-M y c)$ or $\alpha$-Flag antibody. Samples were eluted and processed for immunoblotting. Мyc-EMC3 and ABCA3-Flag immunoprecipitated with each other as well as with EMC1 and EMC4. A weak interaction of calnexin with $A B C A 3-F l a g$, but not with Myc-EMC3 was detected. (D) MLE-15 cells were cotransfected with the same amount of ABCA3-Flag together with control siRNAs or Emc1/Emc3/Emc4 siRNA. Cells were harvested 60 hours after transfection and the lysates were used for immunoblotting. Efficient knockdown of $E m c 3$ and Emc4 decreased ABCA3-Flag and EMC1 proteins. In ( $\mathbf{C}$ and $\mathbf{D}), 3$ independent experiments were performed and representative results are shown. Antibodies used for immunoblotting are indicated on the left of the panels. critical for PC synthesis cause severe and acute lung diseases mediated by AT2 cell injury or surfactant deficiency (1). Misfolding and misprocessing of surfactant proteins cause AT2 cell injury, related in part to the activation of UPR due to the misfolding and accumulation of the abnormal proteins within AT2 cells (1, 2, 14-16). Expression of disease-associated mutant ABCA3 or SP-C proteins in an AT2-like cell line modestly induced expression of EMC3 and EMC4, concomitantly with ER stress (Supplemental Figure 6).

To further interrogate the function of EMC3 in the regulation of pulmonary surfactant protein and lipid homeostasis, we isolated epithelial cells (EpCAM ${ }^{+}$cells) from E18.5 control and Emc3-cKO lungs by magnetic cell sorting (Supplemental Figure 7). EpCAM ${ }^{+}$ cells were then subjected to RNA sequencing, proteomic, and lipidomic analyses. Data from these experiments were integrated and correlated to enable a comprehensive view and quantitative measurements of lipid and protein in AT2 cells, seeking to identify mechanisms by which EMC3 regulates AT2 cell functions. Transcriptional and/or posttranscriptional changes in Emc3-deficient epithelial cells were revealed by integration of transcriptomic and proteomic analyses. Consistent with the presence of misfolded/ misprocessed ABCA3 and surfactant proteins SP-B and SP-C, both proteomic and transcriptomic analyses of Emc3-cKO AT2 cells predicted activation of the UPR signaling pathway (Figure 5, A and B, and Supplemental Table 2). The UPR comprises 3 paral- lel sensing pathways: IRE1 $\alpha / \mathrm{XBP} 1, \mathrm{ATF} 6$, and PERK/eIF2 $\alpha$. The PERK branch of the UPR was activated in Emc3-cKO AT2 cells, as indicated by increased expression of ATF4 (Figure 5, E, F, and I), the major effector of the integrated stress response (ISR) (20), and its downstream target, GADD34 (Figure 5I). In response to PERK-mediated phosphorylation of eIF2 $\alpha$, ATF4 dimerizes with other transcription factors, including ATF3 (Figure 5, C and D) and DDIT3/CHOP, to restore cellular homeostasis or, in the face of prolonged stress, to induce cell death. Likewise, the IRE1 $\alpha$ / XBP1 branch of the UPR was activated by Emc3 deficiency. As a surrogate of IRE1 $\alpha$ activity, splicing of Xbp1 mRNA was increased, as indicated by the increased ratio of spliced $X b p 1(\mathrm{~S})$ to full-length $X b p 1(\mathrm{U})$ (Figure 5J). The functionally active transcription factor $X b p 1$ (S) upregulates a series of chaperones, including HSPA5/BiP and DNAJB9/Erdj4 (Figure 5A and Supplemental Table 2), which are involved in folding of surfactant proteins (21). In contrast to the above 2 branches, ATF6 was not activated, as both the full-length ATF6(P90) and the cleaved transcription factor ATF6(P50) were reduced in Emc3-cKO AT2 cells (Figure 5, G-I). Taken together, our data demonstrated that Emc3 deletion selectively induced UPR pathways in AT2 cells of the murine lung (Figure 5K).

Emc3 deletion disrupts surfactant phospholipid and protein synthesis in AT2 cells. Consistent with accumulation of surfactant proproteins (Figure 3), proteomic analysis of Emc3-cKO AT2 

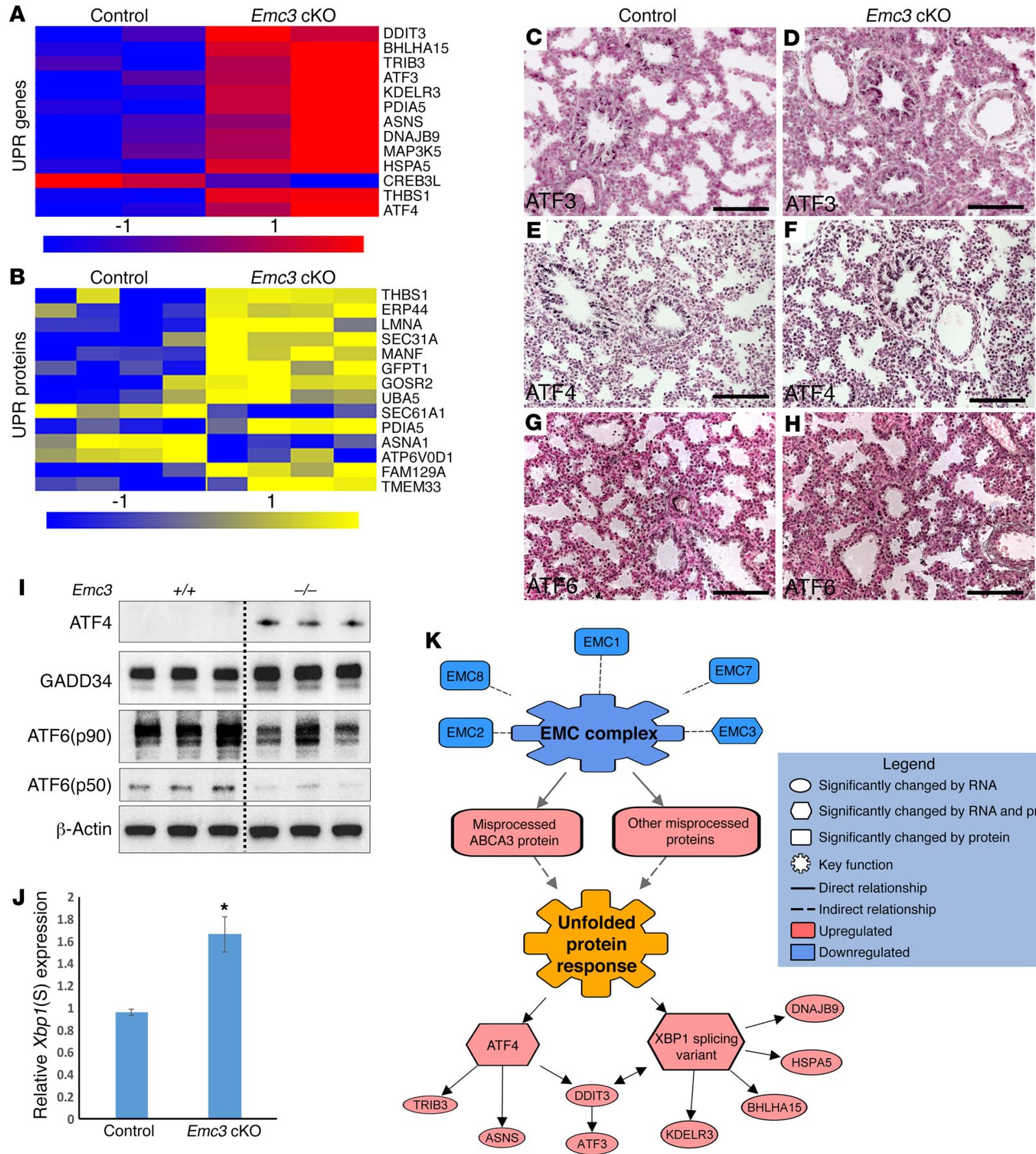

Figure 5. Loss of Emc3 induced the unfolded protein response in EpCAM+ sorted epithelial cells. (A and $\mathbf{B})$ Heatmap of the mRNAs (A, blue/red) and proteins (B, blue/yellow) involved in the UPR pathway are shown. Proteomic and RNA sequencing data were obtained from EpCAM+ sorted epithelial cells from control and Emc3-cKO mice at E18.5. Genes and proteins were categorized by ToppGene. $P$ values and fold changes for each mRNA and protein are listed in Supplemental Table 2. (C-H) Immunohistochemical staining for ATF3 (C and D), ATF4 (E and F), and ATF6 (G and $\mathbf{H}$ ) was performed on lung sections from E18.5 control and Emc3-cKO embryos. ATF3 and ATF4 staining was increased and ATF6 staining was unaltered in the mutant lungs. Scale bars: $100 \mu \mathrm{m}$. (I) Western blots using EpCAM+ cell lysates from control and mutant lungs at E18.5 were performed using the indicated antibodies. (J) Increased Xbp1 splicing in Emc3-cKO mice. Levels of the spliced Xbp1 transcript [Xbp1(S)] were normalized to that of the full-length Xbp1 by qPCR. mRNAs were isolated from E18.5 control and Emc3-cKO EpCAM+ cells. Data are the mean \pm SEM. ${ }^{*} P<0.05$ using unpaired, 2-tailed Student's $t$ test. $n=4 /$ group. (K) Model for the induction of UPR in Emc3-cKO AT2 cells. The model was built based on the integration of RNA sequencing and proteomic data. Relationships between differentially expressed genes and proteins were determined by Genomatix Pathway System (GePS) and Ingenuity Pathway Analysis (IPA) suites. System models were created using IPA's Path Designer. 


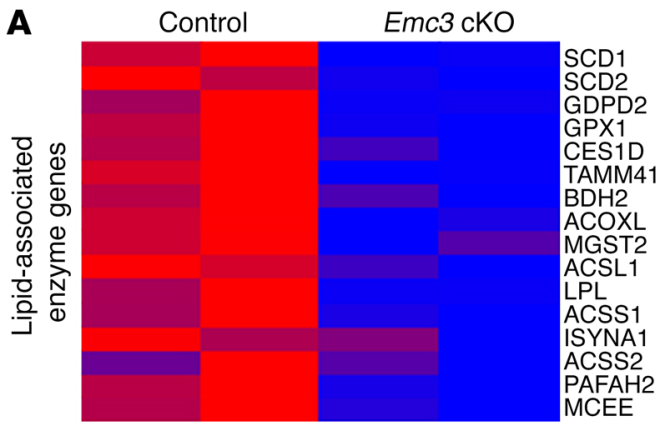

B

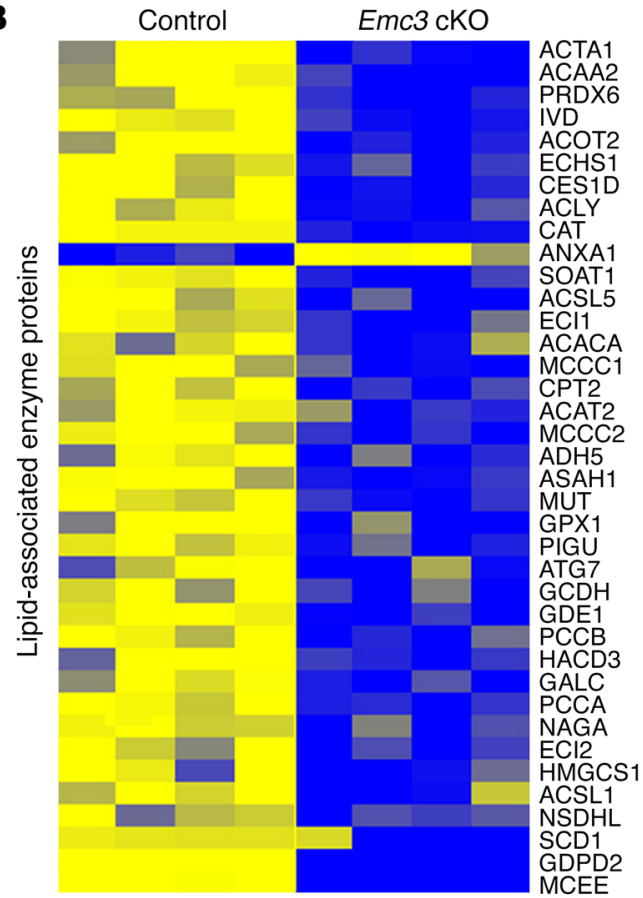

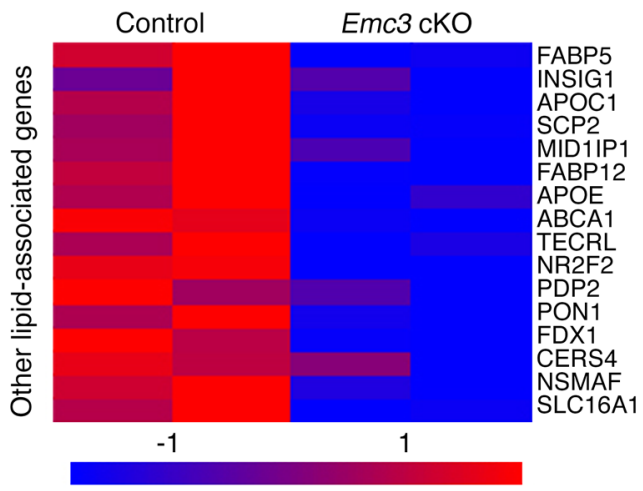

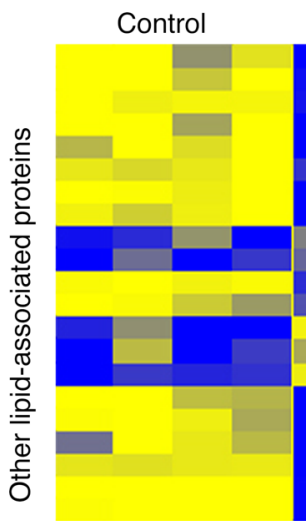

Emc3 cKO

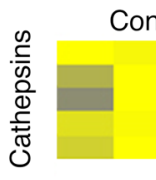

Control

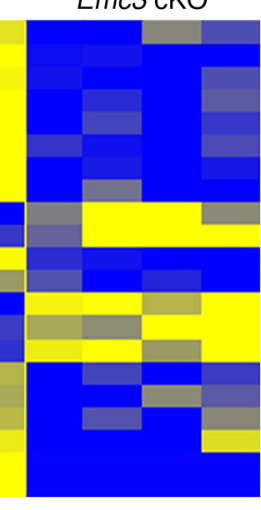

NPC2

$A B C A 3$

SCP2

$A B C D 3$

SFTPB

PC

ATBP5

C3

AKT1

SCPEP1

SORL1

PSAP

EIF6

LAMTOR

ERLIN1

GOLM1

STOML

SLC44A

AGK

Emc3 cKO
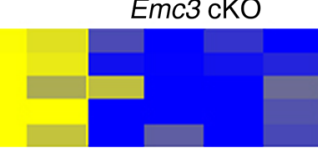

CTSZ CTSB

CTSC

CTSC
Figure 6. Heatmap of the mRNAs and proteins involved in surfactant protein and lipid metabolism. (A and B) RNA

(A, blue/red) and proteomic (B, blue/yellow) sequencing data were obtained from EpCAM+ sorted epithelial cells from control and Emc3-cKO mice at E18.5. Genes and proteins were categorized by ToppGene. $P$ values and fold changes for individual mRNAs and proteins are listed in Supplemental Table 2. cells demonstrated a reduction of a number of proteases critical for the processing of surfactant proteins, including cathepsin $\mathrm{H}$ (CTSH) and napsin (NAPSA) that occurred without changes in their encoding RNAs (Figure 6, A and B). Similarly, levels of other cathepsins, CTSB, CTSC, and CTSZ, were significantly decreased (Figure 6B), which together may reflect altered sorting/trafficking of surfactant proteins to the lysosome and LB compartments.

Besides ABCA3 and UPR components, major groups of proteins were affected at RNA and/or protein levels, including enzymes and transporters involved in lipid homeostasis (Figure 6, A and B, and Supplemental Table 2). Similar to ABCA3 and other EMC subunits, some ER proteins (e.g., ACAT1/2 and ABCD3) were decreased in spite of normal RNA concentrations, indicating that these proteins may be directly or indirectly regulated by EMC3 at the level of protein folding or stabilization. RNAs encoding a number of key enzymes (e.g., PON1, SCD1/2, and ACAT1/2) and lipid transport proteins (e.g., FABP5, SCP2, and ABCD3), some with known roles in surfactant phospholipid synthesis, were decreased (Figure 6A and Supplemental Table 2). Expression of PON1, SCD1/2, FABP5, and SCP2 changed coordinately at both RNA and protein levels, perhaps mediated by loss of SREBP sig- naling in the Emc3-cKO AT2 cells. This change could be due to the transcriptional regulation of active UPR in response to the accumulation of unfolded/misfolded proteins in Emc3-cKO AT2 cells. As a master transcription regulator of cellular lipogenesis, suppression of SREBP1/2 may mediate alterations in surfactant lipid homeostasis $(22,23)$. In Emc3-cKO AT2 cells, lipid concentrations were markedly decreased (Supplemental Table 3). While loss of PG moieties is consistent with defects in Abca3-mutant lungs (18, 24), differential effects among various PC species were observed beyond those changed by ABCA3 deficiency. In contrast to findings in Abca3 gene-targeted mice $(18,24)$, triglycerides accumulated to high levels in Emc3-deficient AT2 cells (Supplemental Table 3). Since triglycerides serve as a source of substrate for surfactant synthesis, their accumulation may indicate the disruption of normal lipid biogenesis in the absence of EMC3.

\section{Discussion}

Since the identification of the EMC complex almost 2 decades ago, our study provides the first evidence to our knowledge that the EMC3/EMC complex is essential in mammalian development. The 3 major defects in Emc3-cKO lung include disrupted surfactant 


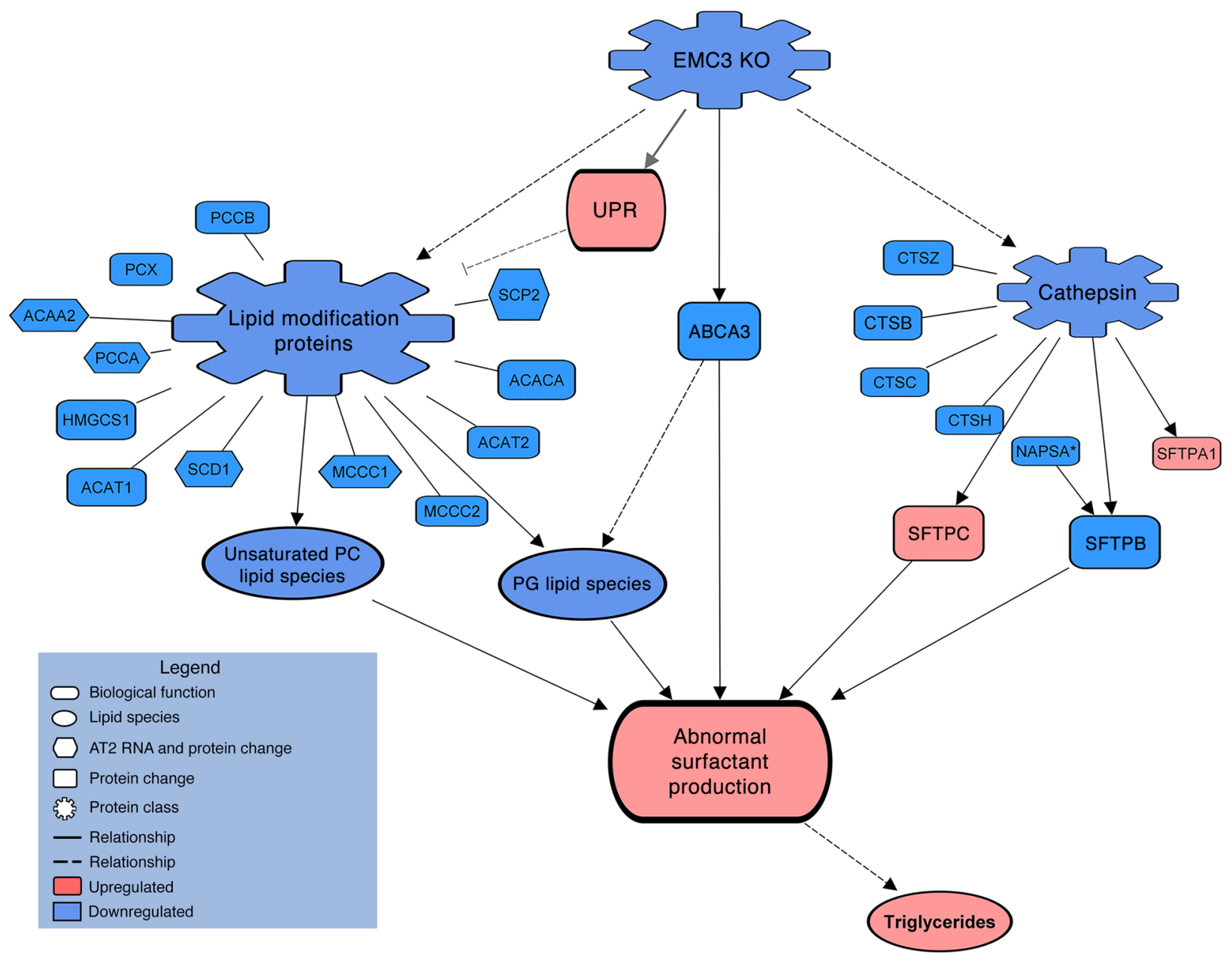

Figure 7. Role of EMC3 in surfactant biogenesis modeled by integration of RNA sequencing and proteomic data. Model for disrupted surfactant production in Emc3-cKO AT2 cells. The model is based on the integration of RNA sequencing and proteomic data. Relationships between differentially expressed genes and proteins were determined by Genomatix Pathway System (GePS) and Ingenuity Pathway Analysis (IPA) suites. System models were created using IPA's Path Designer.

lipid synthesis (Supplemental Table 3), abnormal processing and trafficking of surfactant proteins (Figure 3), and induction of the UPRs (Figure 5), all of which contribute to surfactant deficiency at birth. The production of surfactant by AT2 cells is a highly orchestrated process in which disruption of individual genes causes secondary defects in surfactant lipid and protein homeostasis (1); for example, SFTPB deficiency inhibits packaging of phospholipids into LBs and causes misprocessing of proSP-C. $A B C A 3$ mutations result in failure to transport surfactant lipids into LBs and abnormal routing of surfactant proteins. ABCA3, SFTPB, and SFTPC mutations produce misfolded proteins and induce the UPR, some of which cause AT2 cell injury. The UPR itself may disrupt lipid homeostasis through suppression of SREBP1/2 signaling. Thus, the respiratory failure caused by deletion of Emc3 may reflect combined effects of EMC3 on surfactant production via direct and indirect processes (Figure 7).

While $E m c 3$ deficiency dramatically decreased $A B C A 3$ protein levels, $A b c a 3$-cKO and Emc3-cKO lungs have distinct transcriptional profiles and defects in cellular functions, which is likely due to the more pleiotropic effects of EMC3 on surfactant homeosta- sis. Transcriptional profiling demonstrated that $A b c a 3$ and $E m c 3$ gene-deleted mice shared few changes in their lung mRNA profile (Supplemental Table 1). While loss of ABCA3 compromised the expression of genes related to the biosynthesis and transport of fatty acids and phospholipids, enzymes and transporters primarily involved in lipogenesis were changed at RNA and/or protein levels after deletion of $\operatorname{Emc3}(18,24)$ (Figure 6 and Supplemental Table 2). Moreover, decreased SREBP signaling, perhaps mediated in part by activation of the UPR, may contribute to the inhibition of lipid synthesis in the Emc3-deleted mice. While deletion of Abca3 decreased lung PC and PG content $(18,24)$, the marked increase in triglyceride in Emc3-cKO lungs indicates neutral lipid accumulation and a more general disruption of cellular lipid homeostasis.

EMC3 directly bound ABCA3 and was required for its stabilization in AT2 cells, but also disrupted proteolytic processing and intracellular transport of proSP-B and proSP-C. While EMC3 did not bind to the surfactant proteins, napsin and several cathepsins known to mediate their processing were reduced in the Emc3-deleted mice. Misprocessed surfactant proteins are usually misrouted as has been shown in cultured cells (25), isolated AT2 
cells (26), and in vivo (27), consistent with the abnormal accumulation of surfactant proteins in Emc3-deleted AT2 cells.

Perinatal lung maturation is associated with increasing synthesis of surfactant proteins and lipids required for adaptation to air breathing. AT2 cells are highly metabolic and express high levels of the surfactant-associated proteins and lipids, rendering them sensitive to alterations in surfactant homeostasis that cause ER stress. In the present study, deletion of Emc3 caused misprocessing and accumulation of surfactant proteins and other AT2 cell proteins, as well as accumulation of triglyceride and alterations in lipid concentrations, both impairing AT2 cell functions and probably inducing ER stress. In response to ER stress, UPR pathways are induced to increase ER folding capacity and to maintain cellular homeostasis. The induction of the UPR by the loss of EMC3 and other EMC subunits has been reported in previous findings. In Saccharomyces cerevisiae, deletion of Emc1-6 activated the sensor of misfolded proteins, Ire1p, and induced Hac1p splicing and the UPR (8). In Drosophila, loss of Emc3 in the retina activated IreI/Xbp1 and eIF $2 \alpha$ phosphorylation (12). Consistent with those findings, we found that deletion of Emc3 in AT2 cells activated 2 of the 3 branches of the UPR, including IRE1 $\alpha / \mathrm{XBP} 1$ and PERK/eIF2 $\alpha$, as indicated by $\mathrm{XBP} 1$ and ATF 4 activities, respectively. While the transcription factor XBP1 induces expression of a number of chaperones involved in protein folding and ERAD, PERK inhibits translation initiation and protein synthesis, thereby reducing protein cargoes in the ER. We did not detect the activation of ATF6, a transcription factor that induces UPR target genes, including a number of ER chaperones. During ER stress, activated UPR can interfere with SREBP signaling and thus disrupt lipid metabolism. PPAR $\gamma$ and PPAR $\alpha$, transcriptional targets of XBP1 (28-30), may induce expression of Insig-1/2, thus reducing levels of active $\operatorname{SREBP}(31,32)$. Likewise, ISR activation following eIF $2 \alpha$ phosphorylation reduced active SREBP and the transcription of target genes involved in lipid synthesis (33), providing potential mechanisms by which SREBP signaling is inhibited in the absence of Emc3.

Work in Danio (13), Caenorhabditis elegans $(10,11)$, and Drosophila (12) demonstrated that EMCs are critical for the biogenesis of multipass transmembrane proteins, but not for secreted proteins or type I single-pass transmembrane proteins. Consistent with these findings, our data demonstrated that EMC3 and other EMC subunits directly bound to and stabilized ABCA3, a 12-pass transmembrane protein (Figure 4). While deletion of Emc3 blocked normal processing and trafficking of the secreted protein $\mathrm{SP}-\mathrm{B}$ and the single membrane-spanning protein SP-C (Figure 3), our data suggest that EMC3 does not cause a generalized disruption of protein folding, since deletion of Emc3 in developing lung epithelial cells did not perturb lung growth, morphogenesis, or differentiation. These effects of Emc3 deletion were relatively specific to the processes involved in synthesis and packaging of pulmonary surfactant. Histological, RNA, and proteomic analyses did not reveal widespread disruption of signal transduction, growth, or differentiation. We utilized Shh-Cre (34) to delete Emc3 in the ventral foregut early in the processes of branching morphogenesis. Patterning of the embryonic lung is controlled by diverse signaling pathways mediated by numerous multipass transmembrane proteins, for example, DISP1 and WLS. Preservation of normal lung growth and structure in the Emc3-cKO mice supports a relatively restricted substrate specificity of proteins regulated by the EMC3/EMC complex in AT2 cells.

In summary, our findings support the critical role of EMC3 and the EMC complex that coordinates (a) folding and stability of proteins critical for surfactant function at birth, (b) the UPR in the ER, and (c) regulation of lipogenesis by AT2 cells. EMC3 is a critical component of an ER membrane complex that integrates surfactant protein and lipid synthesis required for air breathing after birth.

\section{Methods}

Mice. To generate the $E m c 3^{f l}$ mice, an embryonic stem cell line containing the knockout-first Emc3 allele (Emc3 $\left.{ }^{\text {tmla(EUCOMM)Wtsi }}\right)$ was purchased from The European Conditional Mouse Mutagenesis Program (EUCOMM) (Figure 1A). After obtaining germ-line transmission, the conditional allele $E m c 3^{f /+}$ was generated and bred with Shh-Cre (The Jackson Laboratory, stock number 005622) for endoderm-specific deletion of $E m c 3$ (Figure 1B). $E m c 3^{f l}$ and $E m c 3^{+}$alleles were genotyped using primers $\mathrm{F}$ (GAGAACTGCTCTGCACACTCC) and R (GTCAGTGCTCTTAACTGCTCTCT). For timed matings, E0.5 was determined by the presence of a copulation plug in the morning. Pregnant dams were sacrificed by $\mathrm{CO}_{2}$ inhalation and embryos were harvested at E18.5. PO newborn pups were sacrificed by anesthesia using a mixture of ketamine, acepromazine, and xylazine and exsanguinated by severing the inferior vena cava and descending aorta. All experiments were performed using at least 4 animals of each genotype per group.

Lung immunohistochemistry, immunofluorescence, and biochemistry. Lungs from E18.5 embryos and P0 newborns were fixed by 4\% paraformaldehyde followed by paraffin embedding. Histological staining, immunohistochemistry, and immunofluorescence were performed on $5-\mu \mathrm{m}$ paraffin sections. Bright-field images were obtained using a Zeiss Axio ImagerA2 microscope equipped with AxioVision Software. Fluorescence images were obtained using a Nikon A1Rsi inverted laser confocal microscope. For the detection of surfactant proteins in embryonic lungs by Western blots, frozen lung lobes were homogenized in phosphate-buffered saline (PBS) supplemented with Protease Inhibitor Cocktail (Sigma-Aldrich); for the detection of ABCA3, frozen lung lobes were homogenized in celLytic M lysis buffer (Sigma-Aldrich) supplemented with the Protease Inhibitor Cocktail; for the detection of factors involved in the UPR, $\mathrm{EpCAM}^{+}$sorted epithelial cells were lysed in RIPA buffer (Thermo Fisher Scientific) supplemented with the Protease Inhibitor Cocktail. The following antibodies were used in this work: rabbit anti-proSP-C (WRAB-9337, Seven Hills Bioreagents); guinea pig anti-proSP-C (generated in the Whitsett laboratory, raised against the N terminus of proSP-C); rabbit anti-mSP-C (WRAB-76694, Seven Hills Bioreagents); rabbit anti-proSP-B (WRAB-55522, Seven Hills Bioreagents); rabbit anti-mSP-B (WRAB-48604, Seven Hills Bioreagents); guinea pig anti-ABCA3 (generated in the Whitsett laboratory, raised against the first loop of $\mathrm{ABCA} 3$ ) (35); rabbit anti-ABCA3 (WRAB-70565, Seven Hills Bioreagents); rat anti-mouse LAMP-1 (1D4B, Developmental Studies Hybridoma Bank); rabbit anti-GAPDH (G9545, Sigma-Aldrich); mouse anti- $\beta$-actin (A5441, Sigma-Aldrich); rabbit anti-Hopx (sc-30216, Santa Cruz Biotechnology); goat antiAGER (AF1145, R\&D Systems); rabbit anti-Flag (F7425, Sigma-Aldrich); mouse anti-Flag (MA1-91878, Thermo Fisher Scientific); mouse antic-Myc (sc-40, Santa Cruz Biotechnology); chicken anti-c-Myc (A-21281, Molecular Probes); rabbit anti-calnexin (SPA-860D 611813, StressGen); mouse anti-PDI (MA3-019, Thermo Fisher Scientific); rabbit 
anti-EMC1 (PA5-23732, Thermo Fisher Scientific); mouse anti-EMC3/ TMEM111 (sc-365903, Santa Cruz Biotechnology); rabbit anti-EMC3/ TMEM111 (HPA042372, Sigma-Aldrich); rabbit anti-EMC4/TMEM85 (ab184162, Abcam); mouse anti-EMC7 (sc-514440, Santa Cruz Biotechnology); rabbit anti-EMC10 (ab181209, Abcam); hamster antipodoplanin (MA5-16113, Developmental Studies Hybridoma Bank); rabbit anti-GRP78/BiP (G9043, Sigma-Aldrich); rabbit anti-NKX2.1 (also known as TTF-1; WRAB-1231, Seven Hills Bioreagents); mouse anti-ATF3 (ab191513, Abcam); rabbit anti-ATF4 (ab184909, Abcam); mouse anti-ATF6 (70B1413.1, Novus); mouse anti-CHOP (2895, Cell Signaling Technology); rabbit anti-GADD34 (PA1-139, Thermo Fisher Scientific); and mouse anti-GFP (sc-9996, Santa Cruz Biotechnology).

Electron microscopy and immunogold labeling. Embryonic mouse lung tissues at E18.5 were fixed and processed using a protocol modified from a previous publication (36). E18.5 mouse lungs were fixed in situ with $2 \%$ paraformaldehyde (Electron Microscopy Sciences, EMS), $2 \%$ glutaraldehyde (EMS), 0.1\% calcium chloride (Sigma-Aldrich), and $0.1 \mathrm{M}$ sodium cacodylate buffer, $\mathrm{pH} 7.3$ (SCB; EMS) overnight at $4^{\circ} \mathrm{C}$. Fixed lungs were cut into 1 - to 2 -mm blocks, postfixed with $1 \%$ osmium tetroxide (EMS) and $1.5 \%$ potassium ferrocyanide (SigmaAldrich) in 0.1 M SCB, pH 7.3, dehydrated in a graded series of alcohol, and infiltrated and embedded with EMbed 812 (EMS). Immunogold labeling was performed as previously described (37). Electron micrographs of lung sections were acquired using a Hitachi TEM H-7650 (Hitachi High Technologies America) with an AMT CCD camera (Advanced Microscopy Techniques).

Morphometric analysis of LBs. To determine whether loss of EMC3 influences LB formation, 40 AT2 cells obtained per animal at E18.5 were randomly selected ( $n=2$ for control and Emc3-cKO mice) for ultrastructural morphometric analysis. Electron micrographs of AT2 cells were acquired at magnification of $\times 5,000$ using a Hitachi $\mathrm{H}-7650$ electron microscope and an AMT TEM camera, followed by manual tracings of LBs in surveyed cells using FIJI, a variant of Image image analysis software. Surface areas and maximal and minimal diameters of traced LBs were estimated. Differences in surface area and diameter between control and Emc3-cKO animals were compared using the Kruskal-Wallis test, where a $P$ value less than 0.05 was considered statistically significant.

Constructs. The C-terminal Myc-DDK-tagged EMC3 construct was purchased from ORIGENE (catalog MR203415). To make the N-terminal Myc-tagged EMC3, Emc3 cDNA was amplified by PCR using primers $\mathrm{F}$ (ttgtcgacCCatggaacaaaaacttatttctgaagaagatctgGCAGGACCAGAGCTGCTGCTTGACTC) and R (ttgtcgacTTAGAATATGGACGTCTGTAGTTCCA) and then subcloned into the pShuttle vector using SalI restriction sites. The construct encoding Flag-tagged mouse ABCA3 was generated by the Whitsett laboratory. Those encoding human SFTPC variants were generated by the Weaver laboratory. The constructs encoding hABCA3-GFP, hABCA3(E292V)-GFP, and hABCA3(L101P)-GFP were provided by Jennifer A. Wambach and Francis S. Cole (Washington University, St. Louis, Missouri, USA).

Cell culture, transfection, and Western blots. MLE-15 cells were cultured as previously described (19). Cell transfection with plasmid and/or siRNA was done using the Lipofectamine 3000 reagent (Life Technologies) following the manufacturer's protocol. Upon harvest, cells were lysed in celLytic M lysis buffer supplemented with Protease Inhibitor Cocktail. Cell debris was removed by centrifugation. For co-IP assays, cells were harvested 48 hours after transfection in lysis buffer
(50 mM Tris-HCl, pH 7.4, 150 mM NaCl, 1 mM EDTA, and 1\% Triton X-100 supplemented with Proteinase Inhibitor Cocktail). Cell lysates were incubated with control beads (the EZview Red Protein G Affinity Gel, Sigma-Aldrich) or anti-Myc beads (Anti-c-Myc Agarose Affinity Gel, Sigma-Aldrich) or anti-Flag beads (ANTI-FLAG M2 Affinity $\mathrm{Gel}$, Sigma-Aldrich) overnight at $4^{\circ} \mathrm{C}$. After removal of the supernatant, immunoprecipitates were recovered from the gel by incubation with Laemmli SDS reducing sample buffer (Bio-Rad) for 20 minutes at $42^{\circ} \mathrm{C}$.

Proteins were resolved by SDS-PAGE using 4\%-12\% gels (NuPAGE Novex gel, Invitrogen) and the MES SDS running buffer (NuPAGE, Invitrogen). Chemiluminescence was observed using the Luminata Western HRP substrate (Millipore) and images were generated using the ChemiDoc Touch imaging system (Bio-Rad) and quantified using Image Lab software (Bio-Rad). For each experiment presented, at least 3 independent experiments were performed and representative results are shown.

The following Silencer Select siRNAs (Thermo Fisher Scientific) were used for transfection: control siRNA (catalog 4457289), Emc1 siRNA 1 (catalog s106663), Emc1 siRNA 2 (s106664), Emc3 siRNA 1 (catalog s82552), Emc3 siRNA 2 (catalog s82553), Emc4 siRNA 1 (cata$\log$ s86111), and Emc4 siRNA 2 (catalog s86112).

Isolation of epithelial cells by magnetic cell sorting. To isolate pulmonary epithelial cells, E18.5 lung lobes were harvested from control and Emc3-cKO embryos and digested with Dispase (Corning) at $37^{\circ} \mathrm{C}$ for 15 minutes. The lobes were transferred to $5 \mathrm{ml}$ of DMEM containing $25 \mathrm{mM}$ HEPES (Gibco) and 120 U of DNAse I (Sigma-Aldrich) and dissociated with a gentleMACS Dissociator (Miltenyi Biotec). The cell suspensions were passed through a $40-\mu \mathrm{m}$ nylon mesh strainer and then pelleted by centrifugation at 300-400 $g$ for 10 minutes. Cell pellets were resuspended in $90 \mu \mathrm{l}$ of autoMACS Running Buffer (Miltenyi Biotec) and 10 $\mu \mathrm{l}$ of CD326 (EpCAM) MicroBeads (Miltenyi Biotec). After incubation for 15 minutes at $4^{\circ} \mathrm{C}, 900 \mu \mathrm{l}$ of autoMACS Running Buffer was added and cells pelleted. After washing twice with autoMACS Running Buffer, cells were resuspended in $500 \mu \mathrm{l}$ of Running Buffer and passed through a 40- $\mu \mathrm{m}$ nylon filter cap into a round-bottom tube prior to sorting using an autoMACS Pro Separator (Miltenyi Biotec). EpCAM-positive (epithelial) and -negative (non-epithelial) cells were isolated. Cells were counted and approximately $5 \times 10^{4}$ EpCAM $^{+}$cells were centrifuged and stained with NKX2.1/proSP-C antibodies and DAPI. Sorting efficiency was determined by the ratio of NKX2.1 $1^{+}$cells (epithelial) and proSP- $\mathrm{C}^{+}$cells (AT2) in all DAPI-stained cells. Results from 4 control and 3 Emc3-cKO mice were quantified using at least 3 independent images per sample.

RNA analyses. Total RNA was isolated from $\mathrm{EpCAM}^{+}$cells or frozen lung lobes using an RNeasy Micro Kit (Qiagen), and reverse transcription reactions were performed using an iScript cDNA synthesis kit (Bio-Rad) following the manufacturers' instructions. Quantitative PCR (qPCR) was performed using a StepOnePlus Real-Time PCR System and TaqMan gene expression assays (Applied Biosystems). Emc3 RNA was detected using Taqman probe Mm01184718_g1 and normalized to the levels of 18S RNA (TaqMan probe 4352930, Applied Biosystems). The levels of the spliced form of XBP1 (TapMan probe Mm03464496_ m1; Applied Biosystems) was normalized to that of the unspliced XBP1 (TapMan probe Mm03464497_s1; Applied Biosystems). Relative expression was calculated using the $\Delta \Delta \mathrm{Ct}$ method and statistical significance was determined by 2-tailed unpaired Student's $t$ test.

$R N A$, protein, and lipid analyses. RNA sequencing was performed on whole-lung RNAs or RNAs extracted from E18.5 lung EpCAM ${ }^{+}$ 
cells. Sequencing and alignment were done by Cincinnati Children's Hospital Medical Center's Gene Expression Core. Emc3-cKO and control samples ( $n=2$ each) were analyzed. Raw reads were aligned using Bioconductor's GenomicAlignment (38). DeSeq was used to analyze the raw gene counts and to calculate differentially expressed genes (39). AltAnalyzer was also used to determine significance (40). Genes that passed only 1 differentially expressed analysis were manually reviewed to determine relevance. All differentially expressed genes had a fold change greater than 1.2, an nbinomTest $P$ value less than 0.05 , and reads per kilobase of transcript per million mapped reads (RPKMs) greater than 1 for both samples in at least one condition (39). The complete data set has been submitted to the NCBI's Gene Expression Omnibus (GEO) database with the accession number GSE90969.

Mass spectrometry-based proteomic and lipidomic analyses were performed at Pacific Northwest National Laboratory on $\mathrm{EpCAM}^{+}$cells isolated from control and Emc3-cKO fetal lungs ( $n=4$ each) at E18.5. Proteomic analyses were performed using tandem mass spectrometry (LC-MS/MS) to profile protein changes employing a label-free relative quantification approach (41). Lipid analyses were performed using LCMS/MS employing a label-free relative quantification approach (42-44). Data were $\log 2$ transformed and median normalized within each sample. Statistically significant changes were determined using 2-tailed, homoscedastic $t$ tests. All significantly changed proteins and lipid species had a $P$ value less than 0.05 and a fold change greater than 1.2. The lipidomic and proteomic data were deposited and are freely available at the MassIVE data repository with the MassIVE ID of MSV000080389 (https://massive.ucsd.edu/ProteoSAFe/static/massive.jsp).

RNAs, proteins, and lipids that were significantly altered were further analyzed using functional genomics tools. ToppGene (https:// toppgene.cchmc.org/) was used to detect functional enrichment of significantly altered RNAs and proteins (45). Genomatix Pathway System (GePS) (www.genomatix.de) and Ingenuity Pathway Analysis (IPA) suites (www.ingenuity.com) were used to determine relationships between differentially expressed genes and proteins based on literature mining. Predicted relationships were then manually reviewed to ensure relevance before being represented in the figures. BRB-ArrayTools was used to generate heatmaps of significant gene and protein changes (46). System models were created using IPA's Path Designer.
Statistics. For qPCR results and quantification of Western blots, values are expressed as the mean \pm SEM. Data were analyzed using a 2-tailed, unpaired Student's $t$ test. $P$ values of 0.05 or less were considered statistically significant. Statistical analyses of RNA, protein, and lipid are described in corresponding sections in the Methods.

Study approval. Mice were housed in pathogen-free conditions according to the protocols approved by the Institutional Animal Care and Use Committee at Cincinnati Children's Hospital Research Foundation.

\section{Author contributions}

$\mathrm{XT}$ and $\mathrm{MH}$ generated the conditional allele of Emc3. XT, XL, and JAW designed experiments and analyzed the data. XT characterized the defects and performed all cell culture experiments. XT and WW examined the unfolded protein response in $\mathrm{EpCAM}^{+}$lung cells by Western blots. JMS and YX analyzed the RNA sequencing, lipidomic, and proteomic data. CLN performed the electron microscopy and immuno-electron microscopy experiments. GC, JEK, EMZ, and CA performed the lipidomic and proteomic analyses. XT, TEW, XL, and JAW wrote the manuscript.

\section{Acknowledgments}

We thank Emily Martin, Karen Apsley, and Samriddha Ray for technical assistance. This work was supported by NIH grants R01HL136722 (to J.A.W. and X.L.) and U01HL134745 and U01HL110964 (to J.A.W.), and grants from the National Natural Science Foundation of China 31571507 and 81361120382 and NIH grant R01GM115995 (to X.L.). Portions of this work were supported by U01HL122703 (to C.A.) and performed in the W. R. Wiley Environmental Molecular Sciences Laboratory (EMSL), a Department of Energy (DOE) office of Biological and Environmental Research (BER) national user facility located at Pacific Northwest National Laboratory (PNNL).

Address correspondence to: Xinhua Lin or Jeffrey A. Whitsett, Cincinnati Children's Hospital Medical Center, 3333 Burnet Avenue, MLC 7029 (X. Lin) MLC 7007 (J.A. Whitsett), Cincinnati, Ohio 45229, USA. Phone: 513.636.2144; Email: xlin@fudan.edu. cn (X. Lin). Phone: 513.803.2790; Email: jeff.whitsett@cchmc. $\operatorname{org}$ (J.A. Whitsett).
1. Whitsett JA, Wert SE, Weaver TE. Diseases of pulmonary surfactant homeostasis. Annu Rev Pathol. 2015;10:371-393.

2. Maguire JA, Mulugeta S, Beers MF. Multiple ways to die: delineation of the unfolded protein response and apoptosis induced by surfactant protein C BRICHOS mutants. Int J Biochem Cell Biol. 2012;44(1):101-112.

3. Wambach JA, et al. Genotype-phenotype correlations for infants and children with ABCA3 deficiency. Am J Respir Crit Care Med. 2014;189(12):1538-1543.

4. Nogee LM. Genetic basis of children's interstitial lung disease. Pediatr Allergy Immunol Pulmonol. 2010;23(1):15-24.

5. Whitsett JA, Wert SE, Weaver TE. Alveolar surfactant homeostasis and the pathogenesis of pulmonary disease. Annu Rev Med. 2010;61:105-119.

6. Mulugeta S, et al. Identification of LBM180, a lamellar body limiting membrane protein of alve- olar type II cells, as the $\mathrm{ABC}$ transporter protein ABCA3. J Biol Chem. 2002;277(25):22147-22155.

7. Yamano G, et al. ABCA3 is a lamellar body membrane protein in human lung alveolar type II cells. FEBS Lett. 2001;508(2):221-225.

8. Jonikas MC, et al. Comprehensive characterization of genes required for protein folding in the endoplasmic reticulum. Science. 2009;323(5922):1693-1697.

9. Christianson JC, et al. Defining human ERAD networks through an integrative mapping strategy. Nat Cell Biol. 2011;14(1):93-105.

10. Richard M, Boulin T, Robert VJ, Richmond JE, Bessereau JL. Biosynthesis of ionotropic acetylcholine receptors requires the evolutionarily conserved ER membrane complex. Proc Natl Acad Sci U S A. 2013;110(11):E1055-E1063.

11. Almedom RB, et al. An ER-resident membrane protein complex regulates nicotinic acetylcholine receptor subunit composition at the synapse.
EMBO J. 2009;28(17):2636-2649.

12. Satoh T, Ohba A, Liu Z, Inagaki T, Satoh AK. $\mathrm{dPob} / \mathrm{EMC}$ is essential for biosynthesis of rhodopsin and other multi-pass membrane proteins in Drosophila photoreceptors. Elife. 2015;4:e06306.

13. Taylor MR, et al. The zebrafish pob gene encodes a novel protein required for survival of red cone photoreceptor cells. Genetics. 2005;170(1):263-273.

14. Hawkins A, et al. A non-BRICHOS SFTPC mutant (SP-CI73T) linked to interstitial lung disease promotes a late block in macroautophagy disrupting cellular proteostasis and mitophagy. Am JPhysiol Lung Cell Mol Physiol. 2015;308(1):L33-L47.

15. Mulugeta S, Nureki S, Beers MF. Lost after translation: insights from pulmonary surfactant for understanding the role of alveolar epithelial dysfunction and cellular quality control in fibrotic lung disease. Am J Physiol Lung Cell Mol Physiol. 2015;309(6):L507-L525. 
16. Tanjore H, Blackwell TS, Lawson WE. Emerging evidence for endoplasmic reticulum stress in the pathogenesis of idiopathic pulmonary fibrosis. Am J Physiol Lung Cell Mol Physiol. 2012;302(8):L721-L729.

17. Clark JC, et al. Targeted disruption of the surfactant protein B gene disrupts surfactant homeostasis, causing respiratory failure in newborn mice. Proc Natl Acad Sci U S A. 1995;92(17):7794-7798.

18. Besnard V, et al. Conditional deletion of Abca3 in alveolar type II cells alters surfactant homeostasis in newborn and adult mice. Am J Physiol Lung Cell Mol Physiol. 2010;298(5):L646-L659.

19. Wikenheiser KA, et al. Production of immortalized distal respiratory epithelial cell lines from surfactant protein $\mathrm{C} /$ simian virus 40 large tumor antigen transgenic mice. Proc Natl Acad Sci U S A. 1993;90(23):11029-11033.

20. Pakos-Zebrucka K, Koryga I, Mnich K, Ljujic M, Samali A, Gorman AM. The integrated stress response. EMBO Rep. 2016;17(10):1374-1395.

21. Dong M, Bridges JP, Apsley K, Xu Y, Weaver TE. ERdj4 and ERdj5 are required for endoplasmic reticulum-associated protein degradation of misfolded surfactant protein C. Mol Biol Cell. 2008;19(6):2620-2630.

22. Bridges JP, et al. Epithelial SCAP/INSIG/SREBP signaling regulates multiple biological processes during perinatal lung maturation. PLOS ONE. 2014;9(5):e91376.

23. Besnard V, et al. Deletion of Scap in alveolar type II cells influences lung lipid homeostasis and identifies a compensatory role for pulmonary lipofibroblasts. J Biol Chem. 2009;284(6):4018-4030.

24. Ban N, et al. ABCA3 as a lipid transporter in pulmonary surfactant biogenesis. J Biol Chem. 2007;282(13):9628-9634.

25. Kabore AF, Wang WJ, Russo SJ, Beers MF. Biosynthesis of surfactant protein C: characterization of aggresome formation by EGFP chimeras containing propeptide mutants lacking conserved cyste- ine residues. JCell Sci. 2001;114(Pt 2):293-302.

26. Beers MF, et al. A nonaggregating surfactant protein C mutant is misdirected to early endosomes and disrupts phospholipid recycling. Traffic. 2011;12(9):1196-1210

27. Wert SE, Whitsett JA, Nogee LM. Genetic disorders of surfactant dysfunction. Pediatr Dev Pathol. 2009;12(4):253-274.

28. Sha H, et al. The IRE1alpha-XBP1 pathway of the unfolded protein response is required for adipogenesis. Cell Metab. 2009;9(6):556-564.

29. Cho YM, et al. X-box binding protein 1 is a novel key regulator of peroxisome proliferator-activated receptor $\gamma 2$. FEBS J. 2014;281(22):5132-5146.

30. Shao M, et al. Hepatic IRE1 $\alpha$ regulates fastinginduced metabolic adaptive programs through the XBP1s-PPAR $\alpha$ axis signalling. Nat Commun. 2014;5:3528.

31. Klopotek A, Hirche F, Eder K. PPAR gamma ligand troglitazone lowers cholesterol synthesis in HepG2 and Caco-2 cells via a reduced concentration of nuclear SREBP-2. Exp Biol Med (Maywood). 2006;231(8):1365-1372.

32. König B, Koch A, Spielmann J, Hilgenfeld C, Stangl GI, Eder K. Activation of PPARalpha lowers synthesis and concentration of cholesterol by reduction of nuclear SREBP-2. Biochem Pharmacol. 2007;73(4):574-585.

33. Harding HP, et al. Bioactive small molecules reveal antagonism between the integrated stress response and sterol-regulated gene expression. Cell Metab. 2005;2(6):361-371.

34. Harris KS, Zhang Z, McManus MT, Harfe BD, Sun X. Dicer function is essential for lung epithelium morphogenesis. Proc Natl Acad Sci US A. 2006;103(7):2208-2213.

35. Stahlman MT, et al. Expression of ABCA3 in developing lung and other tissues. J Histochem Cytochem. 2007;55(1):71-83.

36. Hahn DR, Na CL, Weaver TE. Reserve autophagic capacity in alveolar epithelia provides a repli- cative niche for influenza A virus. Am J Respir Cell Mol Biol. 2014;51(3):400-412.

37. Ridsdale R, Na CL, Xu Y, Greis KD, Weaver T. Comparative proteomic analysis of lung lamellar bodies and lysosome-related organelles. PLOS ONE. 2011;6(1):e16482.

38. Lawrence $\mathrm{M}$, et al. Software for computing and annotating genomic ranges. PLoS Comput Biol. 2013;9(8):e1003118.

39. Anders S, Huber W. Differential expression of RNA-Seq data at the gene level-the DESeq package. University of California, Davis. http:// dmrocke.ucdavis.edu/Class/BST226.2014. Winter/DESeq\%20Pkg.pdf. Accessed September 28, 2017.

40. Emig D, Salomonis N, Baumbach J, Lengauer T, Conklin BR, Albrecht M. AltAnalyze and Domain Graph: analyzing and visualizing exon expression data. Nucleic Acids Res. 2010;38(Web Server issue):W755-W762.

41. Cox J, Mann M. MaxQuant enables high peptide identification rates, individualized p.p.b.-range mass accuracies and proteome-wide protein quantification. Nat Biotechnol. 2008;26(12):1367-1372.

42. Diamond DL, et al. Temporal proteome and lipidome profiles reveal hepatitis $C$ virus-associated reprogramming of hepatocellular metabolism and bioenergetics. PLoS Pathog. 2010;6(1):e1000719.

43. Dautel SE, et al. Lipidomics reveals dramatic lipid compositional changes in the maturing postnatal lung. Sci Rep. 2017;7:40555.

44. Kyle JE, et al. LIQUID: an-open source software for identifying lipids in LC-MS/MS-based lipidomics data. Bioinformatics. 2017;33(11):1744-1746.

45. Chen J, Bardes EE, Aronow BJ, Jegga AG. ToppGene Suite for gene list enrichment analysis and candidate gene prioritization. Nucleic Acids Res. 2009;37(Web Server issue):W305-W311.

46. Simon R, Lam A, Li MC, Ngan M, Menenzes S, Zhao Y. Analysis of gene expression data using BRB-ArrayTools. Cancer Inform. 2007;3:11-17. 